DOI: 10.1136/annrheumdis-2017-eular.4867

\section{OP0184 IMSYC IMMUNOLOGIC SYNOVITIS SCORE: A NEW HISTOLOGICAL SCORE FOR DISCRIMINATING INFLAMMATORY AND NON-INFLAMMATORY ARTHRITIS}

A. Najm ${ }^{1,2}$, B. Le Goff ${ }^{1,2}$, F. Blanchard ${ }^{1}$, J. Amiaud ${ }^{1}$, C. Charrier ${ }^{1}$, V. Krenn ${ }^{3}$. ${ }^{1}$ Inserm UMR 957; ${ }^{2}$ Rheumatology, Nantes University Hospital, Nantes, France; ${ }^{3}$ MVZ-Zentrum für Histologie, Zytologie und Molekulare Diagnostik, Trier, Germany

Background: General Synovitis score (GSS) has been developed by Krenn et al. in order to discriminate inflammatory arthritis (IA) and non-inflammatory arthritis (NIA) (1). This score assesses 3 major components of synovitis: lining layer hyperplasia, activation of resident cells (stroma) and inflammatory infiltrate. All components are graded semi-quantitatively from 0 to 3 and the total score is on 9 . High-grade synovitis is highly associated with IA and is defined by a score upper than 5 with a sensitivity of $61.7 \%$ and a specificity of $96.1 \%$. As immunohistochemistry $(\mathrm{IHC})$ is frequently used to better characterize synovitis, we propose to create a new IMmunologic SYnovitis SCore (IMSYC) adding 5 components to the GSS: CD68, CD3, CD20, CD31 and Ki67 immunostaining.

Objectives: Our work aimed to evaluate the diagnostic performance of this new score including IHC, to define the best cut off for inflammatory arthritis recognition, and to compare its diagnostic performance with the GSS.

Methods: 53 synovial samples from patients were obtained during surgery (arthroplasty or synovectomy). All patients gave written consent prior surgery. Samples were cut and Hematoxylin and eosin stained. CD68, CD3, CD20, CD31 and KI67 Immunohistochemistry were performed. GSS was assessed for each slide and semi-quantitative 4 scale scores (0-3) were given for each immunostaining, in a blind manner. The score is calculated on 24 (GSS 0-9 points, and $0-3$ score for each of the 5 immunostaining). A representative amount of slides was read by 2 observers with a good interobserver variability (spearman correlation coefficient of $0.95, \mathrm{p}<0.0005)$. They then defined a consensual and reproducible scoring atlas.

Results: 53 patients were included. 25 were females $(47,2 \%)$, mean age was 62.1 years [Standard deviation (SD) 13.2 years]. 36 had inflammatory arthritis reparsed as follows: 28 Rheumatoid arthritis (RA), 5 had Psoriatic arthritis, 3 had Undifferentiated arthritis. "Non inflammatory" arthritis group included 10 patients with Osteoarthritis and 7 with ligaments or meniscus injuries. Mean GSS was significantly higher in the IA group 5.70 [SD 0.321] vs.3.51 [SD 0.351]; $\mathrm{p}<0.001$ ). Mean IMSYC was significantly superior in the IA group 14.94 [SD 0.747 ] vs. 8.50 [SD 0.639]; $p<0.001)$. In univariate analysis by logistic regression, GSS (Odd Ratio (OR) 2.27; $p<0.001$ ), CD3 (OR 4.3; $p=0.002$ ), CD68 (OR 4.5; $\mathrm{p}=0.002$ ), Ki67 (OR 11.8; $\mathrm{p}<0.001$ ), and CD31 scores (OR 6.5; $\mathrm{p}=0.001$ ) were significantly associated with IA, however CD20 score was not (OR $0.9 ; p=0.34$ ). ROC curve analysis determined the score of 10.5 out of 24 as the best cut off for discrimination between IA and non-IA with a sensitivity of $74.3 \%$ and specificity of $100 \%$. The area under ROC curves was statistically superior with IMSYC (0.93) compared to GSS (0.81) $(p=0.05)$.

Conclusions: We hereby propose a new synovitis score including IHC. This score has a better sensitivity and specificity than the Global synovitis score for discrimination between IA and non-IA. Moreover, this score accurately describes synovial membrane immunophenotype and could therefore give a basis for tissue driven therapies in rheumatic diseases, especially in RA.

References:

[1] Krenn V et al. Synovitis score: discrimination between chronic low-grade and high-grade synovitis. Histopathology. 2006 Oct;49(4):358-64.

Disclosure of Interest: None declared

DOI: 10.1136/annrheumdis-2017-eular.2547

\section{OP0185 IMMUNOHISTOLOGIC STUDY OF SYNOVITIS FROM PATIENTS WITH UNDIFFERENTIATED ARTHRITIS WHO EVOLVED TO RHEUMATOID ARTRHITIS OR PSORIATIC ARTHRITIS AFTER FOLLOW-UP}

A. Cuervo Aguilera, R. Celis, J. Ramírez, M.V. Hernández, V. Ruíz-Esquide, J. Inciarte-Mundo, R. Sanmartí, J.D. Cañete. Rheumatology, Hospital Clínic Barcelona, Barcelona, Spain

Background: Undifferentiated Arthritis (UA) is defined as an inflammatory oligo/poly arthritis that does not fulfil criteria for a definitive diagnosis.Delay in diagnosis and treatment leads to poor prognosis. Previous studies have found differences in the cellular infiltrate between the synovitis of Rheumatoid Arthritis (RA) and Spondyloarthritis, including psoriatic arthritis (PsA)

Objectives: To identify synovial biomarkers that may be useful to diagnose patients with early UA

Methods: Retrospective longitudinal study.Patients with UA followed in our Arthritis Unit,who underwent arthroscopy between 2000 and 2014.Synovial biopsy were stained by immunohistochemistry with the following antibodies:CD3 for T cells,CD20 for B cells,CD79 for B cells,CD138 for plasma cells,CD31 for vessels,CD68 for macrophages,CD15 for neutrophils,CD117 for mast cells and hsp47 for fibroblasts, and quantified by Digital Image Analysis (Olympus). The same antibodies were evaluated in RA and PSA control groups
Results: 55 UA and 78 controls were included.Table 1 shows the clinical, serological and demographic characteristics.Among patients with UA, $23(42 \%)$ patients met criteria for RA and $32(58 \%)$ for PsA during follow-up.Synovitis of patients with UA had higher macrophage $(C D 68+)$ density in total tissue $(p=0.008)$ and sublining $(S L)(p=0.012)$ than the control group.The UA that evolved to RA had a higher density of CD3 T lymphocytes than the control RA group $(p=0.014)$. No differences were observed in cells of adaptive immunity (CD20 B lymphocytes, CD138 plasma cells), innate immunity (CD117 mast cells, CD15 neutrophils), vessels (CD31) between the 4 groups. The area (\%) stained by anti-hsp47 (synovial fibroblasts) in SL was higher in the RA control group than in the PsA ( $p=0.003)$

Table 1. Data are expressed as mean $\pm S D$

\begin{tabular}{|c|c|c|c|c|c|c|c|}
\hline & $\underset{n=55}{\cup A}$ & $\begin{array}{c}\text { UA-RA } \\
n=23\end{array}$ & $\begin{array}{c}\text { UA-PsA } \\
n=32\end{array}$ & $\mathrm{p}$ & $\begin{array}{c}\mathrm{RA} \\
\mathrm{n}=40\end{array}$ & $\begin{array}{l}\mathrm{PsA} \\
\mathrm{n}=38\end{array}$ & $\mathrm{p}$ \\
\hline Age (years) & $47 \pm 13$ & $51 \pm 13$ & $44 \pm 12$ & 0.058 & $60 \pm 12$ & $54 \pm 13$ & 0.065 \\
\hline Sex (male)n (\%) & $22(40)$ & $6(26)$ & $16(50)$ & 0.074 & $17(43)$ & $23(61)$ & 0.111 \\
\hline Disease duration (years) & $3 \pm 4$ & $3 \pm 2$ & $3 \pm 4$ & 0.114 & $3 \pm 6$ & $2 \pm 2$ & 0.851 \\
\hline Time of follow-up (years) & $7 \pm 4$ & $8 \pm 4$ & $6 \pm 4$ & 0.038 & $7 \pm 3$ & $5 \pm 3$ & 0.008 \\
\hline CRP (mg/dL) & $2.7 \pm 3.9$ & $2.4 \pm 1.9$ & $3.0 \pm 4.9$ & 0.189 & $3.8 \pm 3.2$ & $3.0 \pm 3.8$ & 0.102 \\
\hline DAS28 (ESR) & $4.17 \pm 1.05$ & $4,83 \pm 1,06$ & $3,63 \pm 0,94$ & 0.000 & $5,15 \pm 1,51$ & $4,02 \pm 1,04$ & 0.002 \\
\hline ACPA n (\%) & $4(22)$ & $4(22)$ & $0(0)$ & 0.000 & $30(77)$ & $0(0)$ & 0.000 \\
\hline RF n (\%) & $10(7)$ & $9(41)$ & $1(3)$ & 0.000 & $28(70)$ & $1(3)$ & 0.000 \\
\hline csDMARD n (\%) & $21(41)$ & $7(35)$ & $14(45)$ & 0.472 & $29(73)$ & $19(50)$ & 0.041 \\
\hline bDMARD n (\%) & $2(4)$ & $0(0)$ & $2(6)$ & 0.243 & $8(20)$ & $6(16)$ & 0.628 \\
\hline PDN n (\%) & $7(13)$ & $3(14)$ & $4(13)$ & 0.851 & $11(28)$ & $5(13)$ & 0.117 \\
\hline
\end{tabular}

Conclusions: This is the first immunohistological study of synovitis in a significant group of patients with UA who developed AR or PsA during follow-up. Although there are some differences between the UA and control groups in the density of CD68+ macrophages and lymphocytes T CD3+, these do not appear to be useful for an early diagnosis of UA. On the other hand, unlike the results of some previous studies, we not found differences between the cellular infiltrate (adaptive immunity, innate immunity or vessels) in patients with RA and PsA. The fact that some patients with UA were undergoing treatment prior to synovial biopsy and its retrospective character limit the results of this study

Acknowledgements: Financed: "Fondo de Investigación Sanitaria" (PI14/00785 JD Cañete) del Instituto de Salud Carlos III (ISCIII). Co-financed by BECA FER2015. Premis "Emili Letang", Hospital Clínic. BECA MSD-Sociedad Catalana de Reumatología

Disclosure of Interest: None declared

DOI: 10.1136/annrheumdis-2017-eular.4850

\section{OP0186 TENOFOVIR, A NUCLEOSIDE ANALOG REVERSE TRANSCRIPTASE INHIBITOR FOR TREATMENT OF HIV, PROMOTES OSTEOCLAST DIFFERENTIATION AND BONE LOST IN VIVO IN A MECHANISM DEPENDING ON ATP RELEASE AND ADENOSINE, AND DIPYRIDAMOLE MAY BE A USEFUL TREATMENT TO REVERT THE EFFECTS}

F.M. Conesa ${ }^{1}$, P. Llamas ${ }^{1}$, T. Wilder ${ }^{2}$, P. Atencio ${ }^{3}$, A. Cabello ${ }^{3}$, M. Górgolas $^{3}$, B. Cronstein ${ }^{2}$, R. Largo ${ }^{1}$, G. Herrero-Beaumont ${ }^{1}$, A. Mediero ${ }^{1,2}$. ${ }^{1}$ Joint and Bone Research Unit, lis-Fundacion Jimenez Diaz, Madrid, Spain; ${ }^{2}$ Division of Translational Medicine, Department of Medicine, NYU School of Medicine, New York, United States; ${ }^{3}$ Internal Medicine Department, lis-Fundacion Jimenez Diaz, Madrid, Spain

Background: HIV infection devastates the immune system but also affects other tissues and organs Bone alterations have been observed in HIV disease for nearly two decades, in particular a higher risk of low bone mineral density (BMD) and fragility fractures. Treatment with Tenofovir alone or as part of HAART, leads to changes in bone catabolism markers and significant reductions in BMD in children and young adults. Tenofovir is taken up by cells and phosphorylatedand inhibits HIV-reverse transcriptase by mimicking AMP. We have recently found that Tenofovir inhibits Pannexin-1/Connexin-43-mediated ATP release from cells and decreases extracellular adenosine levels and fibrosis in murine models. Inhibition of osteoclast formation via adenosine $\mathrm{A} 2 \mathrm{~A}$ receptor stimulation or increasing local adenosine concentration stimulates new bone formation as well as rhBMP-2.

Objectives: As adenosine and ATP are key regulators of bone homeostasis, we determined whether Tenofovir directly affects bone by an adenosine- or ATP-dependent mechanism and if treatment with Dipyridamole, an agent that increases extracellular adenosine by blocking cellular adenosine uptake, may be a useful treatment to counteract Tenofovir effects.

Methods: M-CSF/RANKL-induced osteoclast (OC) was studied in primary murine bone marrow culture as the number of TRAP-positive cells after challenge with Tenofovir $(1 \mathrm{nM}-100 \mu \mathrm{M})$ alone or in combination with Dipyridamole $(1 \mathrm{nM}-100 \mu \mathrm{M})$. OC markers were measured by RT-PCR. Pannexin-1 and Connexin-43 expression were permanently knocked down by lentiviral infection with appropriate shRNA or scrambled shRNA and these cells were induced to differentiate into OC by RANKL. Male C57BI/6 mice received Tenofovir $75 \mathrm{mg} / \mathrm{Kg} /$ day alone or in combination with Dipyridamole $1 \mathrm{mg} / \mathrm{Kg} /$ day for 4 weeks. Double labelling of bone with calcein/Alizarin Red to analized bone formation was performed and long bones prepared for microCT and histology.

Results: Tenofovir produced a dose-dependent increase in OC differentia- 
tion $(E C 50=44.5 n M)$ that was reversed by Dipyridamole $(I C 50=0.3 \mu M)$. When Pannexin-1 and Connexin-43 were absent, Tenofovir did not increase OC number. Tenofovir increases Cathepsin K and NFATc1 mRNA levels during OC differentiation, and the effect was reverted by Dipyridamole. Tenofovir reduced bone formation in vivo $(19 \pm 2 \mu \mathrm{m}$ bone aposition vs $35 \pm 4 \mu \mathrm{m}$ untreated $p<0.05)$ and this effect was reverted in the presence of Dipyridamole $(30 \pm 3 \mu \mathrm{m}, \mathrm{p}<0.05$ vs Tenofovir alone). microCT revealed decrease BMD and altered trabecular bone in Tenofovir-treated mice, been reverted in the presence of Dipyridamole. TRAP-staining showed increased OC in vivo in Tenofovir-treated mice $(21 \pm 1$ vs $16 \pm 1 \mathrm{OC} / \mathrm{hpf}$ in untreated, $\mathrm{p}<0.005)$ that was reverted with Dipyridamole. Similar results were obtained for Cathepsin $\mathrm{K}$.

Conclusions: These results indicate that Tenofovir enhances OC differentiation and inhibits osteoblast differentiation by an adenosine-dependent mechanism and suggests that treatment with agents that increase local adenosine concentrations, like Dipyridamole, might prevent bone loss following Tenofovir treatment.

Disclosure of Interest: F. Conesa: None declared, P. Llamas: None declared, T. Wilder: None declared, P. Atencio: None declared, A. Cabello: None declared, M. Górgolas: None declared, B. Cronstein: None declared, R. Largo: None declared, G. Herrero-Beaumont: None declared, A. Mediero Grant/research support from: Instituto de Salud Carlos III, Fondos FEDER

DOI: 10.1136/annrheumdis-2017-eular.3207

\section{OP0187 TRANSGENIC DISRUPTION OF GLUCOCORTICOID-SIGNALING IN MATURE OSTEOBLASTS AND OSTEOCYTES ATTENUATES STRUCTURAL BONE DAMAGE IN A LONG-TERM MURINE K/BXN SERUM-INDUCED ARTHRITIS MODEL}

E. Wiebe ${ }^{1,2}$, C. Spies ${ }^{1,2}$, J. Tu ${ }^{2}$, T. Maleitzke ${ }^{1,2}$, Y. Zhang ${ }^{2}$, M. Seibel ${ }^{2,3}$, H. Zhou ${ }^{2}$, F. Buttgereit ${ }^{4} .{ }^{1}$ Department of Rheumatology and Clinical Immunology, Charité University Hospital, Berlin, Germany; ${ }^{2}$ Bone Research Program, ANZAC Research Institute, University of Sydney; ${ }^{3}$ Department of Endocrinology and Metabolism, Concord Repatriation Hospital, University of Sydney, Sydney, New South Wales, Australia; ${ }^{4}$ Department of Rheumatology and Clinical Immunology, Charité Universitätsmedizin Berlin, Berlin, Germany

Background: The role of endogenous glucocorticoids (GC) in bone metabolism in chronic inflammatory arthritis remains unclear. We have previously shown that disruption of GC-signaling in osteoblasts results in a marked attenuation of arthritis in the $\mathrm{K} / \mathrm{BxN}$ serum-induced and CAIA mouse model with preservation of bone volume and structure ${ }^{1,2}$

Objectives: In order to investigate the impact of endogenous GCs on bone erosion and turnover in chronic inflammatory arthritis, we now studied the effects of disrupted osteoblastic GC-signaling in a long-term murine arthritis model.

Methods: Intracellular GC-signaling in osteoblasts was disrupted by transgenic overexpression of 11 beta-hydroxysteroid dehydrogenase type 2 (11ß-HSD type 2) under the control of a type 1 collagen promoter. Arthritis was induced in 5-week old male transgenic (tg) mice and their wild-type (WT) littermates. In order to maintain a chronically active arthritis, mice were boosted on day 14 and 28 by subcutaneous injection of K/BxN serum, controls (CTR) received PBS, respectively. Severity of arthritis was assessed daily by clinical scoring and ankle size measurements until the endpoint (day 42). Ankle joints were assessed by a histopathologic score and microfocal computed tomography (micro-CT). Systemic effects of inflammation on bone metabolism were quantified by histomorphometry and micro-CT of the tibia.

Results: Acute Arthritis developed in both tg and WT mice and remained active over the period of 42 days, with a reduced, yet non-significant, severity in $\mathrm{tg}$ compared to WT mice. Histological indices of inflammation, cartilage damage and especially bone erosion, additionally assessed by micro-CT, tended to be overall reduced in tg mice, yet not reaching a level of significance. Bone volume and bone turnover did not differ between tg and WT arthritic mice.

Conclusions: The modulating effect of disrupted GC-signaling in osteoblasts in serum-induced autoimmune-arthritis prevails in a chronic inflammatory setting, leading to less severe local inflammation and bone destruction. This supports the important role of endogenous GCs for an intact bone metabolism in inflammatory bone disease.

References:

[1] Tu J, Zhang Y, Kim S, Wiebe E, Spies CM, Buttgereit F, et al. Transgenic Disruption of Glucocorticoid Signaling in Osteoblasts Attenuates Joint Inflammation in Collagen Antibody-Induced Arthritis. Am J Pathol. 2016;186(5):1293-301.

[2] Buttgereit F, Zhou H, Kalak R, Gaber T, Spies CM, Huscher D, et al. Transgenic disruption of glucocorticoid signaling in mature osteoblasts and osteocytes attenuates $\mathrm{K} / \mathrm{BxN}$ mouse serum-induced arthritis in vivo. Arthritis Rheum. 2009;60(7):1998-2007.

Disclosure of Interest: None declared

DOI: 10.1136/annrheumdis-2017-eular.5806
THURSDAY, 15 JUNE 2017

\section{Treat-to-target in axSpA: reality or utopy?}

\section{OP0188 DEFINING CLINICALLY IMPORTANT WORSENING BASED ON ASDAS-CRP FOR AXIAL SPONDYLOARTHRITIS: A DATA-BASED CONSENSUS BY THE ASSESSMENT IN SPONDYLOARTHRITIS INTERNATIONAL SOCIETY (ASAS)}

A. Moltó $^{1}$, L. Gossec ${ }^{2}$, R. Landewé ${ }^{3}$, D. van der Heijde ${ }^{4}$, M. Dougados ${ }^{1}$. ${ }^{1}$ Rheumatology B Department, Cochin Hospital; ${ }^{2}$ Pitié Salpêtrière Hospital, Department of rheumatology, Paris, France; ${ }^{3}$ Department of Clinical Immunology \& Rheumatology, Amsterdam Rheumatology Center, Amsterdam and Zuyderland Medical Center, Heerlen; ${ }^{4}$ Department of Rheumatology, Leiden University Medical Centre, Leiden, Netherlands

Background: Disease flares are increasingly used as outcomes in axial spondyloarthritis (axSpA) trials or observational studies.

Objectives: The objective of this initiative was to define a cutoff for the ASDAS score that best defines the concept of "worsening in axSpA", to be used in the context of clinical trials and longitudinal observational studies.

Methods: Various steps were followed between 2014 and 2017. (a) Initial expert opinion within the steering group to define the scope of the project; (b) systematic literature review to collect cutoffs used for worsening in published studies; (c) vignette-exercise among ASAS members: a theoretical 'paper' patient-vignette, in whom an initial and a final value of an outcome was provided, was judged by the physicians on whether or not the patient had worsened (defining phyworsening) (ref); (d) real-life multicenter international study: data necessary to calculate different outcomes were collected from real patients at 2 consecutive visits (spaced 7 days to 6 months): external standard was defined as a patient's report that he/she had worsened and he/she felt there was a need for treatment intensification. (e) Testing of different changes in the outcomes against both external standards for worsening (phy-worsening and pt-worsening) followed by a consensus and voting procedure among ASAS members in January 2017.

Results: (a) There was consensus about worsening being an absolute change between 2 time-points (without defining time between the 2 time-points) and about exploring cutoffs for 3 outcomes: ASDAS-CRP, BASDAI and pain. (b) The literature review had yielded 27 different cutoffs in 38 studies indicating important heterogeneity (c) The vignette-exercise yielded 12 preliminary definitions for worsening to be tested (as previously reported). (d) In the prospective study the sensitivity and specificity of each cutoff was tested against pt-worsening and judged by the ASAS-community. (e) No consensus was reached for a BASDAI-based definition due to limited performance of all cut-offs, and it was decided to not define a value for a pain-based definition for worsening. Based on aggregated data (Table), a consensus was reached among the ASAS-members to define worsening as a deterioration in ASDAS of at least 0.9 points. While this cutoff led to only moderate sensitivity when tested against pt-worsening, the overall balance of sensitivity and specificity as well as the overall face validity of this cut-off value for ASDAS was deemed most acceptable.

\begin{tabular}{|c|c|c|c|c|}
\hline \multirow[t]{2}{*}{$\begin{array}{l}\text { Cutoff values } \\
\text { for change in } \\
\text { ASDAS }\end{array}$} & \multicolumn{2}{|c|}{$\begin{array}{l}\text { Vignette exercise study } \\
(\mathrm{N}=1150 \text { physician judgments) } \\
\text { against the external standard } \\
\text { 'phy-worsening' (worsening in } \\
\mathrm{N}=591 \text { ) }\end{array}$} & \multicolumn{2}{|c|}{$\begin{array}{l}\text { Prospective real-life study } \\
\text { ( } \mathrm{N}=1169 \text { patients), against the } \\
\text { external standard 'pt-worsening' } \\
\text { (worsening in } \mathrm{N}=127 \text { ) }\end{array}$} \\
\hline & Sensitivity (\%) & Specificity (\%) & Sensitivity (\%) & Specificity (\%) \\
\hline$>=0.6$ & 97 & 65 & 55 & 91 \\
\hline$>=0.9$ & 85 & 87 & 38 & 96 \\
\hline$>=1.1$ & 60 & 94 & 33 & 98 \\
\hline
\end{tabular}

Conclusions: This data-driven ASAS consensus process has allowed to propose an ASDAS-based cutoff value defining worsening in axSpA. As has been observed in other settings, the change defining worsening (at least 0.9 ) is smaller than the change defining improvement which is 1.2 for ASDAS. This definition should now be applied in trials.

References:

[1] Gossec L, et al. Preliminary definitions of 'flare' in axial spondyloarthritis, based on pain, BASDAI and ASDAS-CRP: an ASAS initiative. Ann Rheum Dis. 2016;75:991-6.

Acknowledgements: This study was supported by ASAS.

Disclosure of Interest: None declared

DOI: 10.1136/annrheumdis-2017-eular.4786 